\title{
A novel effective therapeutic approach for treatment of Leishmania tropica through Miltefosine Loaded Chitosan Nanoparticles
}

\author{
Momin Khan \\ Khyber Medical University \\ Muhammad Adnan Shereen \\ Khyber Medical University \\ Mehvish Khokhar \\ Khyber Medical University
}

Atif Kamil ( $\square$ atifkamil@awkum.edu.pk)

Abdul Wali Khan University Mardan

\section{Hazir Rahman}

Abdul Wali Khan University Mardan

Research article

Keywords: Neglected tropical diseases, chitosan, miltefosine, Leishmaniasis, Leishmania tropica

Posted Date: March 21st, 2020

DOI: https://doi.org/10.21203/rs.3.rs-18178/v1

License: (9) This work is licensed under a Creative Commons Attribution 4.0 International License. Read Full License 


\section{Abstract}

Objectives The aim of the present study was to develop miltefosine loaded chitosan nanoparticles (MLCNPs) to enhance the therapeutic efficacy of conventional miltefosine drug and to compare the in vitro activities of both forms of the drug against Leishmania tropica.

Methods MLCNPs were synthesized through ionic gelation with sodium tripolyphosphate (TPP). For the stabilization of nanoparticles, a controlled oxidation of MLCNPs was done with hydrogen peroxide (H2O2). The anti-leishmanial activities of MLCNP were determined by MTT cell viability colorimetric assay. The in vitro hemolysis assay was performed for assessment of toxicity in the human blood.

Results The MLCNPS displayed spherical shape with irregular surface morphology, had a mean size of $200 \mathrm{~nm}$ and a zeta potential of $+10.9 \pm 4 \mathrm{mV}$. A high encapsulation efficacy and drug loading capacity of miltefosine loaded chitosan nanoparticles was also observed. The anti-leishmanial activities of MLCNP were determined by MTT cell viability colorimetric assay. The IC50 value of MLCNPs against promastigote and amastigote were recorded as $0.85 \mu \mathrm{g} / \mathrm{ml}$ and $0.92 \mu \mathrm{g} / \mathrm{ml}$ respectively. MLCNPs proved to be more effective as compared to conventional miltefosine. In terms of toxicity the MLCNPs caused only $2.25 \%$ hemolysis.

Conclusions The engineered MLCNPs could be potential alternative for the treatment of cutaneous leishmaniasis.

\section{Introduction}

Leishmaniasis is vector-borne infectious disease caused by a protozoan parasite belonging to the genus Leishmania. Both anthroponotic and zoonotictransmission of leishmaniasis have been reported. Leishmaniasis is basically a neglected and more likely to be a fatal disease that generally affects under developed and developing countries (Gkolfinopoulou et al., 2013). Leishmaniasis is classified into three basic kinds on the basis of signs and symptoms: cutaneous, mucocutaneous and visceral leishmaniasis (Kheirandish et al., 2013). Each form of the disease is caused by a different species of Leishmania parasite (McGwire and Satoskar, 2013). Leishmaniasis is prevalent worldwide, common in more than 88 countries and it has been evaluated that 0.35 billion individuals are constantly in danger of acquiring the disease (mostly cutaneous and mucocutaneous leishmaniasis) with an expected predominance of 12 million cases and a yearly incidence of 1.5 million cases (Alvar et al., 2012).

Leishmaniasis is the only tropical disease which is treated by non-leishmanial drugs (N. Singh et al., 2012). Treatment choice and clinical representation depends on the type of specie involved in causing Leishmaniasis (Minodier et al., 2007; Murray, 2012). However, treatment procedure and the type of therapy plays a vital role in speeding up the healing process and preventing relapse (Kevric et al., 2015).In the classical leishmaniasis therapy the challenges include approachability of very few drugs, resistance to the accessible drugs, toxicity and lack of cost-effectiveness (De Souza et al., 2010; Desjeux et al., 2004; Launois et al., 2008; Murray et al., 2005). 
Miltefosine belongs to the alkyl-phosphorcholine drugs,that was basically an oral antitumor drug but showed activity against Leishmania as well (Varela et al., 2012). It was registered in India as an effective treatment for visceral and cutaneous leishmaniasis after successful clinical trials (Tiuman et al., 2011). The drug is effective against L. donovani with $97 \%$ healing rate (Freitas-Junior et al., 2012), but more recent reports show a decrease in its efficacy (Soong et al., 2012). For the treatment of leishmaniasis, the dose recommended is $2.5 \mathrm{mg} / \mathrm{kg} /$ day for four weeks (Chrusciak et al., 2011).The conventional miltefosine is linked with some of the adverse effects such as stomach disturbance, intestinal abnormalities including vomiting and nausea. Hepatotoxicity, renal damage and increase in creatinine are some commonly observed side effects. The drug is contraindicated in pregnancy due to its teratogenicity (Khademvatan et al., 2011).

Various nanomedicine formulations such as polymeric nanoparticles, liposomes, nanocapsules and nanoemulsions have been produced by pharmaceutical scientist in order to address the toxicity related issues. Polymeric nanoparticles are one of the extensively used drug delivery systems due to their chemical versatility, additional biocompatibility and simple preparation technique (Chen et al., 2011). They consist of different biodegradable materials like natural or synthetic polymers, metals, or lipids. Nanoparticles are taken up more conveniently by cells than larger micro molecules so they can be used as effective transport and delivery mechanism (Yasinzai et al., 2013). This project was designed to synthesize miltefosine loaded chitosan nanoparticles to enhance its therapeutic efficacy against Leishmania tropica.

\section{Material And Methods}

\subsection{Materials}

The chemicals and media used in this research were purchased from different manufacturer and suppliers. The material used in this study include, Roswell Park Memorial Institute Medium-1640 (RPMI, Lot \# 1868632, GIBCO, USA), Medium 199 (M199, Lot \# CP17-1058, Capricorn Scientific), Heat inactivated Fetal Bovine Serum (hiFBS, Lot \# 10270, GIBCO), Penicillin-Streptomycin solution (PenStrep soln, Lot \# 01161018, Caisson), Trypan blue (Invitrogen, Lot \# 1844453),Chitosan (Sigma-Aldrich), Miltefosine (gifted by local pharmaceutical company), Tripolyphosphate (TPP), D-Trehalose, Sodium hydroxide (Scharlau Chemie S.A), Phosphate Buffered Saline (PBS tabs, Oxoid, Thermo Fisher Scientific), BD Ultra-Fine Insulin Syringes, Geimsa stain, Methanol, Ethanol, Dimethyl Sulfoxide (DMSO).

\subsection{Synthesis of Miltefosine loaded Chitosan Nanoparticles}

Low molecular weight chitosan was interacted ionically with TPP to synthesize the nanoparticles. Nanoparticles were synthesized according to the literature as reported by Bernkop-Schnürch et al., 2006. In brief, chitosan polymer $(0.5 \% \mathrm{w} / \mathrm{v})$ was dissolved in $(1 \% \mathrm{v} / \mathrm{v})$ acetic acid solution. TPP powder $(0.5 \%$ $\mathrm{w} / \mathrm{v})$ was dissolved in distilled water. The $\mathrm{pH}$ was adjusted to 5 and miltefosine $(3 \mathrm{mg} / \mathrm{ml}) \mathrm{was}$ also added to the TPP solution. TPP solution containing miltefosine was added to chitosan solution drop wise 
to synthesize ionically cross-linked nanoparticles. $\mathrm{H}_{2} \mathrm{O}_{2}$ solution $(0.5 \% \mathrm{v} / \mathrm{v})$ was then added to nanoparticles for partial oxidization. The reaction mixture was incubated for one hour under constant stirring at room temperature. To avoid aggregation, D-Trehalose $(3 \% \mathrm{w} / \mathrm{v})$ was consequently added to the nanoparticles solution and centrifuged for $10 \mathrm{~min}$ at $13,400 \mathrm{rpm}$ to accumulate the nanoparticles in pellet.

\subsection{Characterization of miltefosine loaded Chitosan Nanoparticles}

Morphology and size of miltefosine loaded chitosan nanoparticles was determined through scanning electron microscopy (SEM). The magnification used was between $20,000 x$ s to $45,000 x$ s with accelerating voltage of $20 \mathrm{kV}$. Encapsulation efficacy and drug loading contents were determined by colorimetric assay based on the complexation of the zwitterionic miltefosine with the anionic ammonium ferrithiocyanate (NH4Fe[NCS]4) dye developed (Dorlo, Eggelte et al., 2012). The test was derived from an assay developed for the phosphatidylcholine lecithin, in which it forms a coloured complex with ammonium ferrithiocyanate, which can then be extracted inorganic solvents (Dorlo, Hillebrand et al., 2008). This procedure was modified for miltefosine and 1, 2-dichloroethane was used for extraction of the brown-red colored complex. The results were visually and spectrophotometrically assessed at $460 \mathrm{~nm}$. The drug loading of miltefosine in the chitosan nanoparticles was evaluated by determining the difference between the concentration of miltefosine before preparing the nanoparticles and the nonincorporated miltefosine. The following formula was used for drug loading contents in chitosan nanoparticles

Drug loading $\%=$ (Mass of the drug in NP / Mass of NP recovered) $X 100$

The zeta potential of miltefosine loaded chitosan nanoparticles dissolved in deionized water was determined by a PSS Nicomp 380 ZLS zetasizer.

\subsection{Antileishmanial Activities of Miltifosine loaded Chitosan Nanoparticles}

MTT colorimetric assay was used to determine the activity of mitefosine alone and MLCNPs by a method as reported earlier (Dutta et al., 2012). The reagent enters the live cells and goes into their mitochondria where it is reduced to an insoluble formazan product, thus a dark purple color is obtained in the wells. No change in color is seen if the cell density is non-viable. Stock solutions of Miltefosine loaded chitosan nanoparticles and conventional miltefosine were prepared. Preparation procedure was same for both compounds in a way that $1 \mathrm{mg}$ of drug (compound) was dissolved in $1 \mathrm{ml}$ of distilled water to get the final concentration of $1000 \mu \mathrm{g} / 1000 \mu \mathrm{l}$. MTT reagent is soluble in water therefore the solution is prepared by dissolving $1 \mathrm{mg}$ reagent in $1 \mathrm{~mL}$ distilled water. Cultured media containing $1 \times 10^{7}$ parasites per $\mathrm{ml}$ was used to carry out the assay.

In a 96-well plate under sterile culture conditions, axenically grown $100 \mu$ l promastigotes were plated. Each of synthesized nanoparticles and conventional drug solution was added up to the volume of $100 \mu \mathrm{l}$ to initial well and serially diluted by transferring $100 \mu$ l of solution up to last well. $100 \mu l$ was discarded 
from last well, to keep the balance. $10 \mu \mathrm{l}$ of MTT reagent was added to each well after 72 hours of incubation at $24{ }^{\circ} \mathrm{C}$. As MTT is light sensitive so the plate was wrapped in aluminium foil and further incubated for 4 hours at $24{ }^{\circ} \mathrm{C}$ after adding the reagent and then centrifuged at $3000 \mathrm{rpm}$ for 3 minutes. The supernatant was discarded from wells and the pellet was diluted with $100 \mu \mathrm{l}$ of DMSO to stop the enzymatic reaction. The wells were incubated again for 1 hour in shaking incubator at $24{ }^{0} \mathrm{C}$. Absorbance was checked at an optical density of $570 \mathrm{~nm}$ by a microplate reader. As a positive control, media containing parasites was added to wells without any drug. And for negative control only media without parasites and drug was added to wells. The experiment in duplicate was performed for the observation of sensitivity to each drug. The same procedure was performed amastigote with two modification i.e. the $\mathrm{pH}$ of the medium was reduced to 5 and then grown on $37^{\circ} \mathrm{C}$. The data obtained from microplate reader was then subjected to Graphpad prism version 5 software for statistical analysis.

\subsection{In vitro toxicity study of Miltifosine loaded Chitosan Nanoparticles}

In vitro Hemolysis assay was performed to check the toxicity of drug in the blood. It evaluates hemoglobin discharge in the plasma due to certain release of drug, which serves as an indicator of red blood cell lyses. First $3 \mathrm{~mL}$ of blood was collected from healthy volunteer to perform hemolysis assay. The blood was immediately centrifuged at $1500 \mathrm{rpm}$ for 15 minutes to prevent clotting. To attain erythrocytes, plasma and the white buffy layer in the form of supernatant was discarded carefully by aspiration with a pipette. The pellet containing erythrocytes were then washed for three times with $1 \mathrm{X}$ PBS for 5 minutes. The erythrocytes suspension was prepared by mixing $11 \mathrm{~mL}$ of $1 X$ PBS into $3 \mathrm{~mL}$ of centrifuged erythrocytes. The drug solutions of synthesized nanoparticles and conventional drug were prepared in distilled water $(100 \mu \mathrm{g} / \mathrm{mL}$ each). $100 \mu \mathrm{l}$ of erythrocytes suspension was added to $1 \mathrm{~mL}$ of the nanoparticles and conventional drug solution each. Both the reaction mixtures were incubated for 4 hours at $37 \mathrm{OC}$. Lastly Eppendorf tubes were centrifuged at 13,000 rpm for 15 minutes and the remaining hemoglobin in supernatant was measured by spectrophotometer at $570 \mathrm{~nm} .1 \mathrm{~mL}$ of PBS was used as the negative control with $0 \%$ hemolysis, and $1 \mathrm{~mL}$ triton-X 100 was used as the positive control with approximately $100 \%$ hemolysis. The percentage hemolysis was calculated as follows

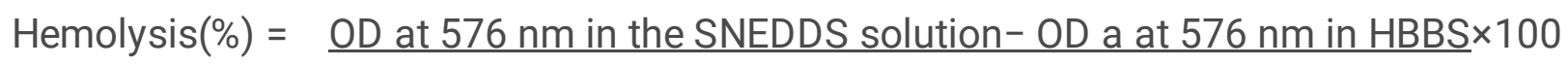

OD at $576 \mathrm{~nm}$ in $0.1 \%$ Triton X-100 - OD at $576 \mathrm{~nm}$ in HBBS

\section{Results}

\subsection{Synthesis and accumulation of Miltifosine loaded Chitosan Nanoparticles}

Miltefosine loaded thiolated nanoparticles were prepared according to the literature previously described (Bernkop-Schnurch et al., 2006). At the end of the experiment milky color solution of NPs were observed (Fig.1) and by centrifugation at 13400rpm for 10 minutes the miltefosine loaded chitosan nanoparticles 
were collected in the pellet form and then by the help of lyophilizer the NPs were lyophilized at $-30^{\circ} \mathrm{C}$ and $0.01 \mathrm{mbar}$ pressure for further use in the experimrnt.

\subsection{Characterization of Miltifosine loaded Chitosan Nanoparticles}

The size and surface morphology of miltefosine loaded thiolated nanoparticles were determined using scanning electron microscopy. The diameters of nanoparticles were ranging from 200 to $300 \mathrm{~nm}$ but average diameter $250 \mathrm{~nm}$. The miltefosine loaded chitosan nanoparticles displayed spherical shape with irregular surface morphology (Fig. 2) and no aggregation was observed. Drug loading content of miltefosine in nanoparticles were determined was $91.5 \mu \mathrm{g} / \mathrm{ml}$ ( Table 1). The miltefosine loaded thiolated nanoparticles possessed a zeta potential of (positive) $+10.9 \pm 4 \mathrm{mV}$ (Table 1).

\subsection{Antileishmanial Activities of Miltifosine loaded chitosan Nanoparticles}

In-vitro anti-leishmanial activity was carried out in 96 well microtitter plates as previously described. Antipromastigote and anti-amastigote activities were performed against Leishmania tropica by MTT colorimetric method. The $\mathrm{IC}_{50}$ was evaluated by a software Graph pad Prism version 5.04 and found that the activity of miltefosine loaded chitosan nanoparticles was higher than the conventional miltefosine. The anti-promastigote activity $\left(\mathrm{IC}_{50}\right.$ value) of conventional miltefosine, chitosanand miltefosine loaded chitosan nanoparticles (MLCNPs) were $0.15 \mu \mathrm{g} / \mathrm{ml}, 0.32 \mu \mathrm{g} / \mathrm{ml}$ and $0.07 \mu \mathrm{g} / \mathrm{ml}$ respectively (Table 2). The anti-amastigote activity in terms of $\mathrm{IC}_{50}$ value of conventional miltefosine, chitosan and MLCNPs were $0.18 \mu \mathrm{g} / \mathrm{ml}, 0.381 \mu \mathrm{g} / \mathrm{ml}$ and $0.09 \mu \mathrm{g} / \mathrm{ml}$.

\subsection{Toxicity studies of Miltifosine loaded chitosan Nanoparticles}

Conventional miltefosine showed more hemolysis than MLTNPs, as the light pink color of its supernatant was observed in the tube. PBS was used as negative control and transparent supernatant was clearly seen in the tube which showed that no hemolysis occurred there. The last tube containing Triton-X 100 (positive control) showed dark red color due to complete breakdown of erythrocytes.It was observed that less hemolysis was induced by synthesized nanoparticle (2.25\%) as compared to conventional miltefosine (4.76\%). PBS shows $0 \%$ hemolysis where as Triton-X 100 implies potent hemolytic activity with $100 \%$ hemolysis (Table 3 ).

\section{Discussions}

The aim of this research project was to synthesize Miltefosine loaded Chitosan Nanoparticles in order to increase the therapeutic potential of mitifosine in order to minimize its toxicities and increase their antileishmanial activities. These MLCNPs were synthesized by ionic gelation with TPP as reported earlier by Tiyaboonchai in 2003. Chitosan based nanoparticles are broadly synthesized using the same technique (Tiyaboonchai, 2003). The first reported Chitosan nanoparticles preparation by ionic gelation method was done in 1997 in Spain (Calvo et al., 1997). Result of our study revealed the average size of MLCNPs is $250 \mathrm{~nm}$ with spherical shape, irregular surface morphology and no aggregation. Studies showed that 
chitosan nanoparticles synthesized by this method mentioned above are small in size ranging from 200 $\mathrm{nm}$ to $300 \mathrm{~nm}$ in diameter (Liu et al., 2008; Makhlof et al., 2010). The drug loading content of MLTNPs was found to be $91.5 \mu \mathrm{g} / \mathrm{ml}$. This high amount of DLC was noticed because of the zwitterionic and amphiphilic nature of the drug used in the experiment (Thagele et al., 2011; Dorlo et al., 2012). The surface charge is another essential feature for the nanoparticles attachment to cell surfaces. Positive charge zeta potential of nanoparticles when interacts with negative charge mucus layer results in strong electrostatic interaction. The MLCNPs showed a zeta potential of $+10.9 \pm 4 \mathrm{mV}$. In 2016 , the study was done on preparation of liposomal nanoparticles, the zeta potential observed was $-8.2 \pm 3.50 \mathrm{mV}$. It indicated that zeta potential of liposomal formulation is slightly less than MLCNPs (Ribeiro et al., 2016).

In vitro, MTT colorimetric assay was performed for synthesized nanoparticles against $L$. tropica. In this study biological activity of MLTNPs and conventional miltefosine was assessed against leishmaniasis. The $\mathrm{IC}_{50}$ value calculated for MLCNPs promastigotes of Leishmania tropica was $0.07 \mu \mathrm{g} / \mathrm{ml}$ which is significantly lower than un modified miltefosine $\left(\mathrm{IC}_{50}=0.15 \mu \mathrm{g} / \mathrm{ml}\right.$. In 2012 , a study carried out in Iraq to calculate the $\mathrm{IC}_{50}$ value of miltefosine showed to be $0.19 \mu \mathrm{g} / \mathrm{ml}$ which means that $\mathrm{IC}_{50}$ value calculated in ours experiment was almost the same as their study (Ali, 2012). It is indicated clearly that the efficacy of MLCNPs was higher than the conventional miltefosine. The satisfying result of MLCNPs could be due to the antimicrobial characteristic of chitosan as we also observed anti-leishmanial activity of chitosan alone which $\mathrm{s} \mathrm{IC}_{50} 0.32 \mu \mathrm{g} / \mathrm{ml}$. It may be considered a synergistic effect of both the drug (miltefosine) and polymer (chitosan) which turned out into successful outcome. It has been reported that chitosan acquires not only antiparasitic property but has good activity against bacteria and fungi, which is why this polymer has been the center of attention for many researchers (Hafdani and Sadeghinia, 2011). In 2017, a study carried out in Brazil revealed the antileishmanial activity of chitosan which opened a path for drug development. Scientific researchers could use chitosan polymers as a pharamacological tool for treating leishmaniasis that lessen the required dose as well as the side effects caused by conventional drug (Lima et al., 2017)

The drugs contain chemical compounds that may induce a hemolytic effect and many drugs have severe adverse effects on erythrocytes. In this experimentation, hemolysis assay was performed to examine the hemolytic activity of synthesized nanoparticles. It was observed that less hemolysis was induced by synthesized nanoparticle (2.25\%) as compared to conventional miltefosine (4.76 \%). In 2016, a similar study was carried out where hemolytic activity of miltefosine was checked, considerable hemolysis of $5.4 \%$ was observed (Alonso and Alonso, 2016) which is similar to our results. Hence, less synthesized nanoparticle showed less hemolysis as compared to conventional miltefosine. Synthesized nanoparticles did not show significant hemolysis so they could be utilized and evaluated for leishmaniasis therapy in animal models (Chávez-Fumagalli et al., 2015).

\section{Conclusions}

Ant-leishmanial activities of the miltefosine can be enhanced significantly by modifying and incorporating this drug into chitosan nanoparticles. Miltefosne loaded chitosan nanoparticles may 
provide better treatment regime compared to conventional unmodified miltefosine.

\section{Declarations}

\section{Acknowledgements}

We are thankful to the office of research innovation and commercialization (ORIC) Khyber Medical University, Peshawar for partially sponsoring the research project.

\section{Conflict of Interest}

All the authors contributed in this manuscript has no financial or academic conflict of interest.

\section{Funding Source}

The office of research innovation and commercialization (ORIC), Khyber Medical University, Peshawar provided partially funding for accomplishment of this research project.

\section{Ethical Approval}

This study has been approved by the bioethical committee of the Department of Microbiology, Institute of Basic Medical Sciences, Khyber Medical University, Peshawar, KPK, Pakistan.

\section{References}

Ali ZH. Cytotoxicity of Miltefosine Against Leishmania Major Promastigotes. Adv Biores 2012; 3 (4): 90 94.

Alonso $\mathrm{L}$ and Alonso A. Hemolytic Potential of Miltefosine is Dependent on Cell Concentration: Implications for In Vitro Cell Cytotoxicity Assays and Pharmacokinetic Data. Biochim Biophys Acta Biomembr 2016; 1858: 1160-1164.

Alvar J, Velez ID, Bern C, Herrero M, Desjeux P, Cano J, et al. Leishmaniasis Worldwide and Global Estimates of its Incidence. PloS One 2012; 7: 35671.

Bernkop-Schnürch A, Weithaler A, Albrecht K, Greimel A. Thiomers: Preparation and In Vitro Evaluation of A Mucoadhesive Nanoparticulate Drug Delivery System. Int J Pharm 2006; 317 (1): 76-81.

Calvo P, Remuñán-López C, Vila-Jato JL, Alonso M. Novel Hydrophilic Chitosan-Polyethylene Oxide Nanoparticles as Protein Carriers. J Appl Polym Sci. 1997; 63: 125-132.

Chávez-Fumagalli MA, Ribeiro TG, Castilho RO, Fernandes SOA., Cardoso VN, Coelho CSP, et al. New Delivery Systems for Amphotericin B Applied to the Improvement of Leishmaniasis Treatment. Rev Soc Bras Med Trop 2015; 48 (3): 235-242. 
Chen M, Wang S, Tan M, Wang Y. Applications of Nanoparticles in Herbal Medicine: Zedoary Turmeric Oil and Its Active Compound $\beta$-elemene. Am J Chin Med 2011; 39 (6): 1093-1102.

Chrusciak-Talhari A, Dietze R, Talhari CC, Da-Silva RM, Yamashita EPG, De-Oliveira PG, et al. Randomized Controlled Clinical Trial to Access Efficacy and Safety of Miltefosine in the Treatment of Cutaneous Leishmaniasis Caused by Leishmania (Viannia) Guyanensis in Manaus, Brazil. Am J Trop Med Hyg 2011; 84 (2): $255-260$.

Desjeuxp. Leishmaniasis: Current Situation and New Perspectives. Comp Immunol Microbiol Infect Dis 2004; 27: 305-318.

DeSouza MC, De-Assis EA, Gomes RS, Marques-Da SEA, Melo MN, Fietto JLR, et al. The Influence of EctoNucleotidases on leishmaniaamazonensis Infection and Immune Response in C57b/6 Mice. Acta Trop 2010; 115: 262-269.

Dorlo TP, Eggelte TA, De-Vries PJ, Beijnen JH. Characterization and Identification of Suspected Counterfeit Miltefosine Capsules. Analyst 2012; 137 (5): 1265-1274.

Dutta A, Bandyopadhyay S, Mandal C, Chatterjee M. Development of a Modified MTT Assay for Screening Antimonial Resistant Field Isolates of Indian Visceral Leishmaniasis. Parasitol Int 2005; 54 (2): 119-122.

Freitas-Junior LH, Chatelain E, Kim HA, Siqueira-Neto JL. Visceral leishmaniasis treatment: What do we have, what do we need and how to deliver it? Int J Parasitol Drugs Drug Resist 2012; 2:11-9.

Gkolfinopoulou K, Bitsolas, N, Patrinos S, Veneti L, Marka A, Dougas G et al. Epidemiology of Human Leishmaniasis in Greece, 1981-2011. Euro Surveill 2013; 18 (29): 20532.

Hafdani FN and Sadeghinia N. A Review on Application of Chitosan as a Natural Antimicrobial. World Acad Sci Eng Technol 2011; 50: 252-256.

Kevric I, Cappel MA, Keeling JH. New World and Old World Leishmania Infections: A Practical Review. Dermatol Clin 2015; 33: 579-593.

Khademvatan S, Gharavi MJ, Rahim F, Saki J. Miltefosine-Induced Apoptotic Cell Death On Leishmania Major And L. Tropica Strains. Korean J Parasitol 2011; 49 (1): 17-23.

Kheirandish F, Sharafi AC, Kazemi B, Mohebali M, Sarlak A, Tarahi MJ, et al. Identification of Leishmania Species using PCR Assay on Giemsa-Stained Slides Prepared From Cutaneous Leishmaniasis Patients. Iran J Parasitol 2013; 8: 382.

Launois p, Tacchini CF, Kienym P. Cutaneous Leishmaniasis: Progress Towards a Vaccine. Expert Rev Vaccines 2008; 7: 1277-12871650-1662. 
Lima DDS, Gullon B, Cardelle-Cobas A, Brito LM, Rodrigues KA, Quelemes PV, et al. Chitosan-Based Silver Nanoparticles: A Study of the Antibacterial, Antileishmanial and Cytotoxic Effects. J Bioact Compat Polym 2017; 32: 397-410.

Liu Z, Jiao Y, Wang Y, Zhou C, Zhang Z. Polysaccharides-Based Nanoparticles as Drug Delivery Systems. Adv Drug Deliv Rev 2008; 60 (15): 1650-1662.

Makhlof A, Werle M, Tozuka Y, Takeuchi H. Nanoparticles of Glycol Chitosan and its Thiolated Derivative Significantly Improved the Pulmonary Delivery of Calcitonin. Int J Pharm 2010; 397: 92-95.

Mcgwire B \& Satoskar A. Leishmaniasis: Clinical Syndromes and Treatment. Qjm 2013; 107: 7-14.

Minodier P and Parola P. Cutaneous Leishmaniasis Treatment. Travel Med Infect Dis 2007; 5: $150-18$.

Murray HW, Berman JD, Davies CR, Saravia NG. Advances in Leishmaniasis. Lancet 2005; 366: 15611577.

Ribeiro JB.P, Miranda-Vilela AL, Graziani D, De-Aguiar GMR, Amorim AAS, Garcia RD, et al. Evaluation of the Efficacy of Systemic Miltefosine Associated with Photodynamic Therapy With Liposomal Chloroaluminium Phthalocyanine in the Treatment of Cutaneous Leishmaniasis Caused by Leishmania (L.) Amazonensis in C57bl/6 Mice. Photodiagnosis Photodyn Ther 2016; 13: 282-290.

Singh N, Kumar M, Singh RK. Leishmaniasis: Current Status of Available Drugs and New Potential Drug Targets. Asian Pac J Trop Med 2012; 5 (6): 485-497.

Soong L, Henard, CA, Melby PC. Immunopathogenesis of Non-Healing American Cutaneous Leishmaniasis and Progressive Visceral Leishmaniasis. Semin Immunopathol 2012; 34 (6): $735-751$.

Tiuman TS, Santos AO, Ueda-Nakamura T, Dias-Filho BP, Nakamura CV. Recent Advances an Leishmaniasis Treatment. Int J Infect Dis 2011, 15 (8), 525-532.

Tiyaboonchai W. Chitosan Nanoparticles: A Promising System for Drug Delivery. Nare Uni J $2003 ; 11$ (3): 51-66.

Varela-M RE, Villa-Pulgarin JA, Yepes E, Müller I, Modolell M, Muñoz DL, et al. In Vitro and In Vivo Efficacy ff Ether Lipid Edelfosine against Leishmania Spp. And SbV-Resistant Parasites. PLoS Negl Trop Dis 2012; $6(4): 1612$.

Yasinzai M, Khan M, Akhtar N, Shahnaz G. Drug Resistance in Leishmaniasis: Current Drug -Delivery Systems and Future Perspectives. Future Med Chem 2013; 5 (15): 1877-1888.

\section{Tables}

Due to technical limitations, tables are only available as a download in the supplemental files section. 


\section{Figures}
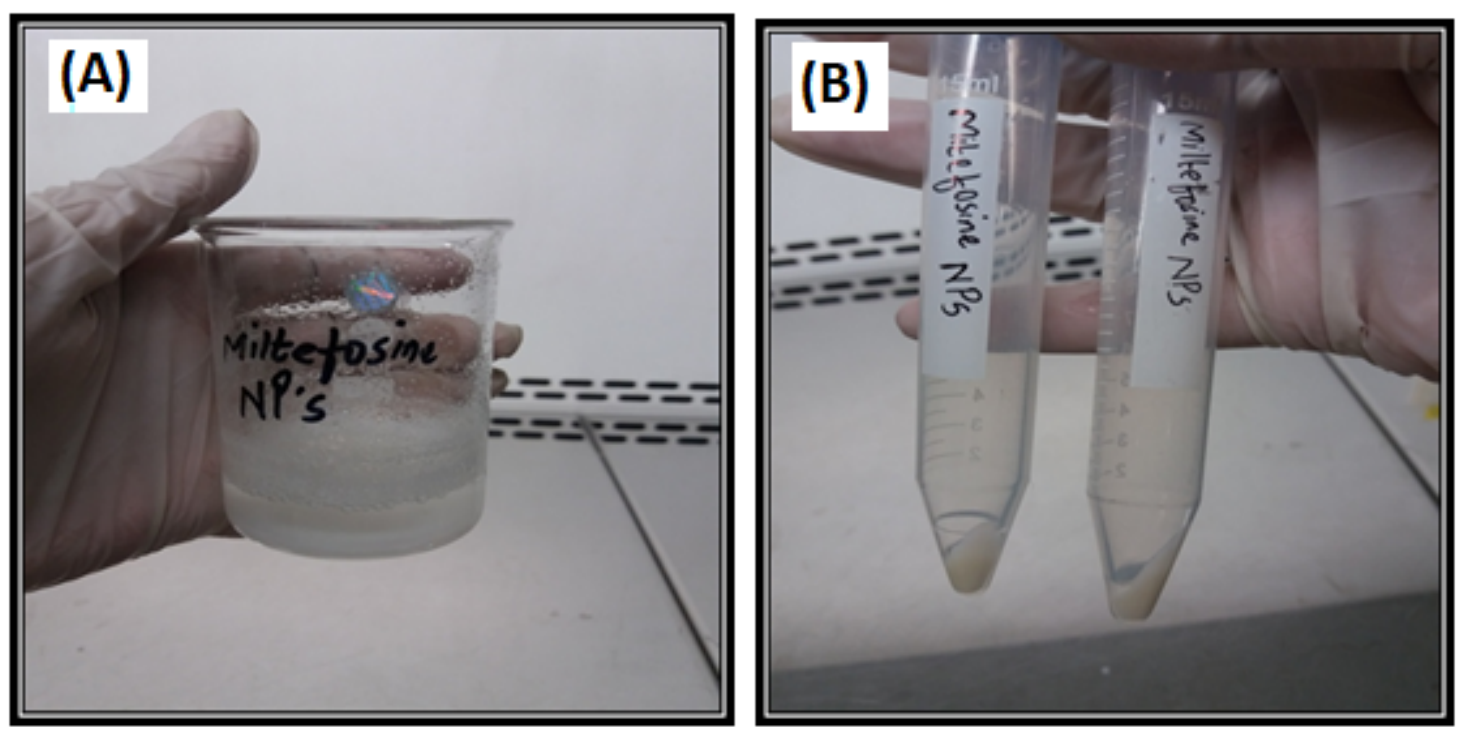

Figure 1

The milky solution and pellet form of MLCNPs. The chitosan polymer, TPP powder and miltefosine solutions were mixed for formation of ionically cross-linked nanoparticles. H2O2 and D-Trehalose were added before centrifugation. The resulting milky solution (A) and pellet (B) MLCNPs, after centrifugation are shown.
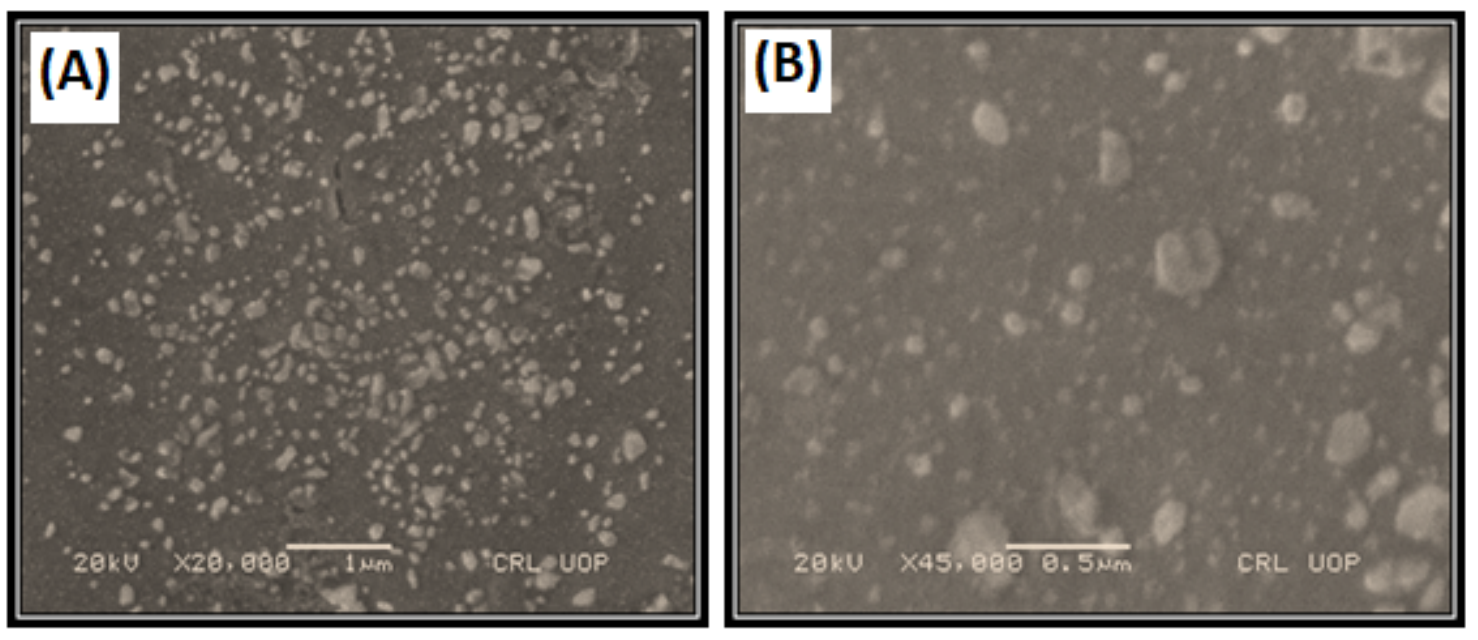

\section{Figure 2}

Morphological examination of MLCNPs. The morphology and size of Miltefosine Loaded Chitosan Canoparticles was determined through scanning electron microscopy (SEM). The magnification used was between 20,000xs (A) to 45,000xs (B) with accelerating voltage of $20 \mathrm{kV}$. The MLCNPs displayed spherical shape with irregular surface morphology. 


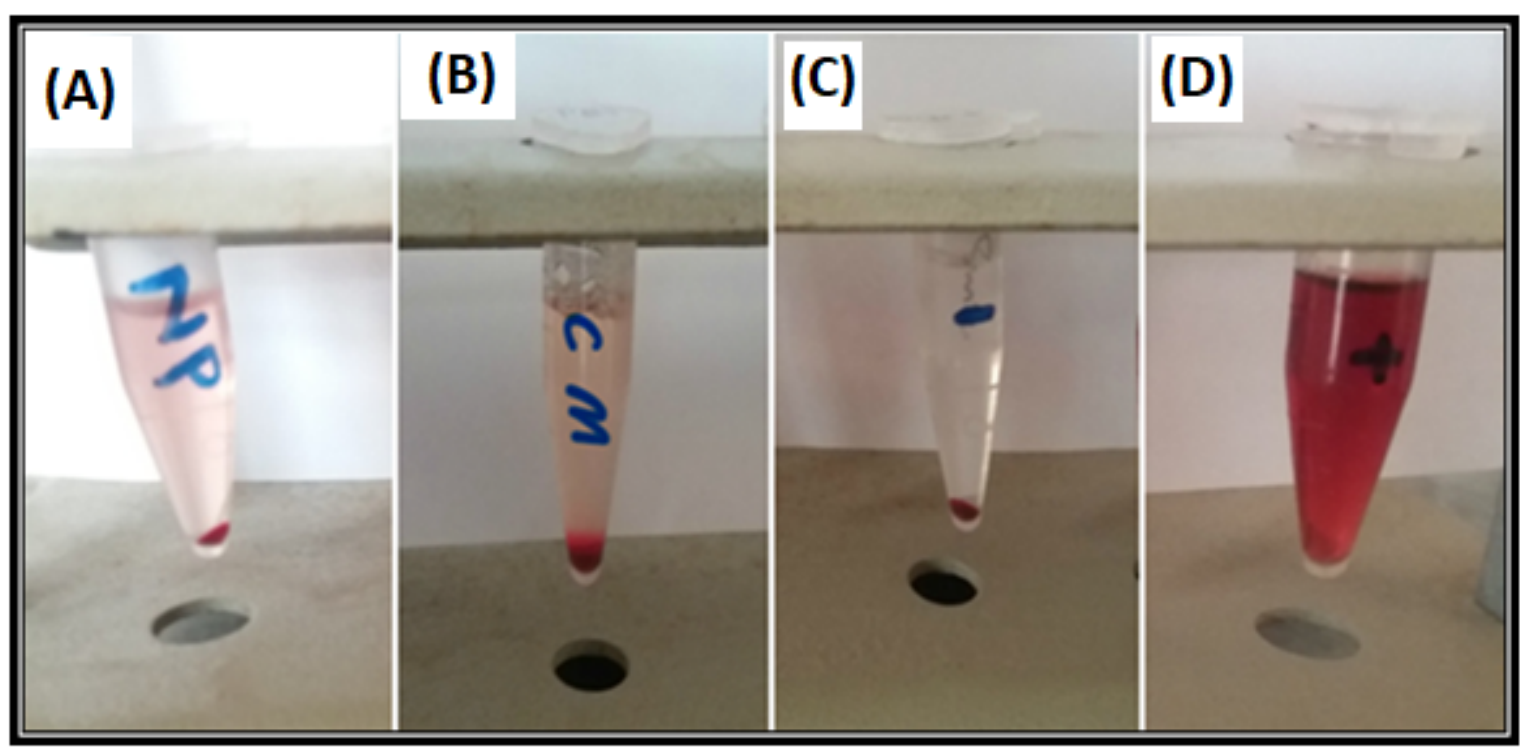

\section{Figure 3}

In vitro hemolysis assay for Mitefosine and MLCNPs. In vitro hemolysis assay was performed to check the toxicity of drug in human blood. The human blood was exposed to each drug and the remaining hemoglobin in supernatant was measured. Conventional miltefosine showed more hemolysis (B) with light pink color, than MLTNPs (A), the negative control PBS solution (C) is transparent (no hemolysis) and the positive control Triton-X 100 solution (D) showed dark red color due to erythrocytes lysis.

\section{Supplementary Files}

This is a list of supplementary files associated with this preprint. Click to download.

- Table3.tif

- Table2.tif

- Table1.tif

- FiguresandTablesLegends.docx 